\title{
HUMANISMO DE PERTENENCIA AL MUNDO DE LA VIDA Y CULTURA SOSTENIBLE
}

\author{
HUMANISM OF BELONGING TO THE WORLD OF SUSTAINABLE \\ LIFE AND CULTURE
}

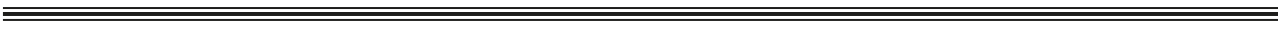

Joaquín García Carrasco ${ }^{a}$ y Macarena Donoso González ${ }^{b^{*}}$
\end{abstract}

Fechas de recepción y aceptación: 26 de febrero de 2021 y 24 de marzo de 2021

DOI: https://doi.org/10.46583/edetania_2021.59.822

Resumen: Observamos en la cultura contemporánea una metamorfosis de la alfabetización y el desdibujo de las fronteras de los campos de conocimiento: son características de la Nueva Ilustración, guiada por preguntas transversales y puntos de vista interdisciplinares. La educación constituye el aglutinador más importante de las Humanidades y el punto desde el que valorar la sostenibilidad de la cultura. La especie humana necesita la cooperación sociocultural para vivir; esta innovación biocomportamental implicó el reciclamiento de las redes neuronales, para poder vivir con lucidez. La cultura evolucionó desde una convicción humana de dominio sobre el mundo de la vida (humanismo de dominancia). La historia demostró que la vida humana es dependiente de la calidad de los ecosistemas (humanismo de dependencia). Hoy, necesitamos vivir en la convicción de que pertenecemos al mundo de la vida (humanismo de pertenencia) y de que somos responsables de su sostenibilidad y de su calidad.

Palabras clave: cultura, alfabetización, Ilustración, humanismo, responsabilidad.

\footnotetext{
${ }^{a}$ Catedrático de Universidad. Departamento de Teoría e Historia de la Educación. Universidad de Salamanca.

${ }^{\mathrm{b}}$ Departamento de Educación. Universidad Antonio de Nebrija.

${ }^{*}$ Correspondencia: Universidad Antonio de Nebrija. Facultad de Lenguas y Educación. Calle Santa Cruz de Marcenado 27. 28015, Madrid, España.

E-mail: mdonoso@nebrija.es
} 
Abstract: In contemporary culture, we observe a metamorphosis of literacy and the blurring of the boundaries of the fields of knowledge: they are characteristics of the New Enlightenment, guided by transversal questions and interdisciplinary points of view. Education constitutes the most important unifier of the Humanities and the point from which to value the sustainability of culture. The human species needs sociocultural cooperation to live; this biobehavioral innovation involved the recycling of neural networks to live lucidly. Culture evolved from a human conviction of dominance over the world of life (dominance humanism). History proved that human life is dependent on the quality of ecosystems (dependency humanism). Today, we need to live in the conviction that we belong to the world of life (humanism of belonging) and that we are responsible for its sustainability and quality.

Keywords: culture, literacy, Enlightenment, humanism, responsibility.

\section{INTRODUCCIÓN: METAMORFOSIS DE LA ALFABETIZACIÓN Y DE LOS CAMPOS DE CONOCIMIENTO}

Hoy, el concepto de alfabetización ha sufrido una metamorfosis. Ha pasado a significar el proceso de introducción al conocimiento, manejando muchos mediadores: la palabra, la lectura y la escritura (el trato con los libros) y el manejo de las tecnologías de la comunicación y la información. La palabra alfabetización se presenta calificada de múltiples maneras: alfabetización matemática, alfabetización biológica, alfabetización digital... La metamorfosis de la alfabetización corre pareja con los cambios de límites en los campos de conocimiento.

Actualmente, el interés no se cifra en el "objeto de conocimiento", sino en la complejidad del problema. Indagar en un problema, sobre todo humano, obliga, sin remedio, a la interdisciplinariedad. La metamorfosis de la alfabetización y la caída de límites en los campos de conocimiento difumina la arquitectura de las disciplinas. Esta constelación de cambios en la manera de pensar ha sido denominada la Nueva Ilustración (Sánchez Ron, 2011).

Parece que la teoría de la educación académica, un área de conocimiento reconocida, también se ve afectada por esos cambios. Hace años, nadie dudaba respecto a la relevancia de las perspectivas biológicas en el estudio de problemas fundamentales de la educación de los seres humanos. Hoy, solo queda una cátedra en la Universidad Autónoma de Barcelona con el título Biología de la Educación. ¿Qué será de ella con estos vientos que soplan? Y su cate- 
drático titular, el doctor J. M. Asensio, ya está jubilado. La transversalidad de los conocimientos puede ser un problema para teorías de andar seguros, pero es un requisito indiscutible en la práctica de la incorporación cultural. En la actualidad, muchos piensan que "gente de cultura" es gente que cultiva en campos de letras. Esta es una de las manifestaciones de la brecha que separa las ciencias de las humanidades.

Si algo nos ha quedado evidente, después de unos seis millones de años de evolución, es que el Gran Simio que somos tiene como destino biológico, como su dominio vital, conseguir cultura para vivir, cultura por necesidad vital. Hoy, muchas mujeres mueren por una cultura insostenible en las relaciones emocionales; otros andan con hábitos de consumo perjudiciales como consecuencia de una cultura corporal insostenible; el mantenimiento de la salubridad de la vida se torna problemática, porque andamos con una cultura vital insostenible.

Lo que hoy se denomina Educación Ambiental plantea más un punto de vista que un programa de disciplina académica; no puede reducirse a una especie de reconciliación con la planta y con el animal; es una manera de llevar a cabo la lectura del mundo de la vida, como corresponde al animal humano, que, para saber vivir, tiene que aprender a hacerlo.

\section{LA ALFABETIZACIÓN COMO PRÁCTICA DE LUCIDEZ EN LA LECTURA DE LA VIDA}

Si hubiésemos aprendido a leer las tramas del mundo de la vida no estaríamos, ahora, temiendo por la biodiversidad, ni urgiendo el sentimiento de responsabilidad hacia ella. Podríamos describir la cultura como el proceso de hacer legible el dominio vital: los seres humanos tienen que aprenderlo. Al final, más que un acto de creación, la cultura trata de un acto de enseñanza; un acto de cultivo, como su propio nombre indica. Lo que mejor muestra la pertenencia a la condición humana, desde el principio y por principio, no es tanto el acto de invención, sino el acto de cooperación, el que comparte manera de vivir. La crianza, como se decía antes, es el acto de cooperación por excelencia. El gran invento, la iniciativa más sorprendente, la práctica de cooperación más asombrosa que se observa en el reino animal es la cultura humana.

Stanislas Dehaene es un neurocientífico francés, de especial interés para el dominio de la pedagogía, por haber puesto el foco de atención en la investiga- 
ción de las bases neuronales de la conciencia y en los correlatos neuronales de la práctica de la lectura. De formación inicial matemática, redirigió su interés investigador tras la lectura del libro L'homme neuronal de J. P. Changeux (1986). Dirige hoy el Laboratorio de Neuroimagen Cognitiva (Cognitive Neuroimaging $\mathrm{Lab})^{1}$. Lo consideramos un ejemplo de cómo desde la neurociencia puede enriquecerse la teoría de la educación. Sin duda, una de las cuestiones principales de esa teoría, es la que interroga sobre "¿Cómo aprendemos?”; desde la neurociencia podría interpretarse como los "talentos del cerebro" (Bermúdez de Castro, 2010); estos problemas son precisamente los que centran la investigación de S. Dehaene (2019).

Este es uno de los autores que nos confirman en la idea de que "educación" es el concepto con mayor poder de integración de las humanidades; como el de "evolución" es el de mayor poder aglutinador de toda la biología (Dobzhansky, 1937). S. Dehaene, en otro libro reciente, se pregunta para qué sirve la conciencia. Responde el autor que la conciencia nos permite llevar a cabo cadenas de operaciones cuidadosamente controladas; estas integraciones controladas, mejoradas por el lenguaje, que nos permite conversaciones y discursos, son esenciales para compartir y distribuir nuestros pensamientos a través de una trama social. Es decir, que el papel esencial de la conciencia sería contribuir a la acción cultural, hacer posible la cooperación que contribuye a la formación de la mente de otras personas. Si tenemos razón, nunca como ahora, la educación se sitúa en el centro de la deliberación humanista.

Todas las demás especies animales aprenden, pero solo en las especies Homo apareció como patrón ecológico diferencial enseñar a vivir en el mundo de la vida. J. Mosterín, en un precioso libro titulado Ciencia viva, abre el prólogo afirmando: "Vivir bien es, entre otras cosas, vivir despierto, vivir con los ojos abiertos, darse cuenta de quiénes somos y dónde estamos, practicar la virtud de la lucidez" (Mosterín, 2001, 13).

Enseñar empezó siendo únicamente una acción intencionada, cuando los homínidos no habían aprendido a hablar; se comunicaban mediante comportamientos, acciones y gestos, la esencia de los verbos, pero sin palabras. El diferencial específico de la evolución humana fue que, de una u otra forma,

${ }^{1} \mathrm{http} / / /$ www.unicog.org/?page_id=6. Con una producción científica muy importante. 
las mentes aprendieron a comprender la intencionalidad de otros y, por eso, pudieron tener la intención de ayudarles. Ahora tomamos la acción de leer como metafórica para toda acción de comprensión: lectura de la mente de otros.

La alfabetización es como prender la luz, como caminar con alguien llevando una luz encendida, alumbrar, y, en el mismo acto, enseñar a ver, mientras se está leyendo para aprender. Cuando se consigue que la mirada de alguien se fascine con algún fenómeno del mundo o, simplemente, con alguna de las cosas de la vida, se la está enseñando a leer. La alfabetización es, hoy, proceso necesario para beneficiarse de la legibilidad del mundo.

De ahí que un problema interesante de antropología es el de tratar de explicar cómo tuvo lugar el reciclamiento del cerebro primate. Evolucionó para tratar con acontecimientos del mundo y sus indicios. ¿Cómo se recicló para que pudiera tratar con los signos y símbolos de la comunicación oral y escrita? Este tema lo ha investigado S. Dehaene, indagando sobre "las neuronas de la lectura", como en dos tiempos: en primera instancia, mediante técnicas de neuroimagen de nueva generación, investiga el comportamiento neuronal mientras las personas leen; en segunda instancia, infiere de esa investigación criterios científicos para orientar el aprendizaje de la lectura y sus métodos de enseñanza (Dehaene, 2014).

En la actualidad, la comprensión del ser humano y su papel en el mundo de la vida, trabajo para la antropología, es al mismo tiempo un proceso de alfabetización humanista y un proceso de alfabetización biológica, para que pueda ser un motor de cultura sostenible. En realidad, la novedad bioteológica que introdujeron las especies Homo, sobre todo cuando aparecieron los Sapiens hablando, fue que la vida de los seres humanos, por la cultura, habría de transformarse en un gigantesco problema pedagógico.

\section{La CUlTURA BusCó Un PUESTO PARA EL HOMBRE EN EL COSMOS}

El siglo XIX generó una metafórica de los escalafones y de posiciones de los seres vivos en el cosmos. El microscopio había sido el instrumento adecuado para vislumbrar en lo muy pequeño y el telescopio para indagar en lo infinitamente lejano; los macroscopios son los instrumentos con los que acometemos la investigación de las complejidades. Macroscopios son nuestros puntos 
de vista, nuestras perspectivas, nuestros modelos, nuestras teorías, nuestras convicciones, nuestras creencias, nuestros prejuicios. A ellos nos aferramos como a clavos, porque sin ellos no es posible la comprensión de los grandes paquetes de significado, aunque los tratemos como si fuesen cosas: la vida, el universo, el ser humano, la sociedad, siempre lo conocemos en perspectiva, desde nuestros personales macroscopios (De Rosnay, 1977).

\subsection{El presentimiento de no ser el centro de nada}

Ya pasa de quinientos años el tiempo en que una contingencia cambió la imagen de la posición de los humanos en el universo. Hasta ese momento, siguiendo a Tolomeo, imaginábamos que la Tierra estaba en el centro de todo, la propia retina era el centro de giro de todo. Copérnico empezó a escribir en 1503 su tratado De revolutionibus orbium celestium; las contradicciones de la vieja imaginería eran tan abrumadoras, que no quedaba otra que situar al Sol en el centro de nuestro universo. Era tan consciente Copérnico, como su editor Osiander, de que el torpedo se dirigía a la línea de flotación del barco de las convenciones, que el autor demoró desde 1530 a 1543 la publicación. La tesis insinuada en el libro era que la morada de los humanos no estaba en el centro de nada; lo mismo que arriesgó Galileo. Giordano Bruno sacó las conclusiones: en cuestiones de puesto, un humano no es más que una hormiga ante el infinito universo, afirmación que le llevó a la pira.

En el fondo, la discusión por la posición equivalía a justificar el predominio de la iniciativa de los seres humanos sobre las vidas de todos los demás seres vivos. De ello no se deducía sino predominio, no derivaba en un principio de responsabilidad (Jonas, 1995). La meta de todos los seres vivos, por lo visto, era satisfacer las necesidades de los únicos que son racionales.

Apenas tranquilizada la tormenta provocada por Copérnico, cuando otra nueva contingencia cultural vino a revolver en la caja fuerte de las seguridades, dando a entender que no éramos el centro. 


\subsection{El puesto del hombre está entre los seres genuinamente animales}

El 23 de noviembre de 1859, un día antes de que apareciera en las librerías El origen de las especies de Darwin, Thomas Henry Huxley, profesor de zoología en Londres, amigo personal de Darwin y defensor de su propuesta teórica sobre la evolución, le envió una carta en la que afirmaba: “...estoy dispuesto a ir a la pira, si es necesario...", en defensa de la opinión de que natura non facit saltum (la naturaleza no da saltos) (Darwin, 2003); la vida en su recorrido temporal no ha dado brincos en el vacío dejando bordes de abismos que no pudieran saldarse. H. Huxley más decidido, si cabe, que el propio Darwin en incluir al hombre en el proceso de la evolución, editó en 1896 el libro Man's Place in Nature, 'el puesto del hombre en la naturaleza', para profesar su convicción de que los seres humanos son un eslabón más en la cadena de la vida. El puesto del hombre en el Cosmos también fue el título de una obra de Max Scheler, firmada en Frankfurt a finales de abril de 1928 (Scheler, 1979).

El atractivo extraordinario de las tesis de Darwin era que tenemos origen animal; se dejaba entender que el hombre era un animal genuino. A pesar de todo, en el fondo, todos se debatían entre las dos caras innegables que mostraba la especie humana. De un lado, era irrefutable que la denominación Homo de Linneo hacía referencia a un grupo de mamíferos vertebrados que caminan sobre dos patas; pero, por otro lado, se identificaban caracteres que se oponían a la clasificación de animal en general; este era Sapiens.

Este problema "de las dos caras" se enraizaba en una solución dualista cartesiana de una res cogitans y una res extensa. En el ser humano conviven, según esta propuesta, dos realidades: una material o de carne, donde acontecen los procesos biológicos, y otra inmaterial, donde transcurren los procesos de conocimiento. Hoy, aquel problema se analiza intentando superar dificultades de argumentación muy exigentes dentro de una propuesta de "unidad psicosomática" (Damasio, 1996); en este caso el problema se formula habitualmente como el de las "relaciones mente-cuerpo": ya no se trata de dos realidades, sino de una que vive y piensa. ¿Cómo podemos pensar siendo corporales, cómo es posible este estar en el mundo "de cuerpo pensante"? 


\section{Ser en el mundo de CUerpo Pensante}

Hoy, la coincidencia es alta, por un lado, la línea argumental evolutiva del parentesco biológico en todo el mundo de la vida, por otro, la del carácter excepcional del modo de vida humano. Creemos que, entre el "sí" y el "pero", fue excavándose la garganta que separó las "dos culturas", la de las humanidades y la de las ciencias (Snow, 1977). A veces, se piensa que el objetivo y valor del pensamiento científico se expresa y cifra en "el dominio de la naturaleza" (Bataner, 2001), por aquello de que, también aquí, quien tiene la información tiene el poder; y quien no parece tener límite en el conocimiento sobre el mundo tampoco encuentra límite para su poder sobre el mundo. El conocimiento sobre la interconexión de todo el mundo de la vida no solo desvela la interdependencia de todas las formas de vida, tanto en el tiempo como en el espacio, sino que se instituye en foco de una advertencia moral: ¡Cuidado, no todo lo que nos es accesible se encuentra impunemente disponible!

\subsection{Del lugar que asignemos a los humanos en el mundo de la vida depende lo que atendamos en su formación}

La última revisión del puesto del ser humano en el mundo consistió en mostrar que todo el mundo de la vida y el de las comunidades humanas se encontraban en el mismo espacio moral. El principio de responsabilidad moral y la deliberación en la que nos hacemos responsables ha de abarcar todo el mundo de la vida. Los humanos no pueden vivir sin pensamientos sobre el mundo; la interpretación del mundo proporciona contenido imprescindible para el desarrollo de la mente, es su mentalidad; en tales materiales es donde está encarnada la mente y, de todos ellos obtiene la tea con la que enciende la conciencia; ni la mente ni la conciencia son recipientes previos al propio proceso del pensamiento o de la experiencia. La mente y la conciencia se realizan y emergen en nuestro modo de vivir, inician en la experiencia su andadura y mueren con ella, cuando la experiencia de la vida se apaga.

Es curioso que precisamente la cultura, el instrumento fundamental de que disponen los humanos para la visión del mundo, se constituya al mismo tiempo en la principal fuente para los embotamientos mentales. 
No es posible plantear la pregunta sobre lo que hacemos aquí ni construir la respuesta, sin asumir un punto de vista, una perspectiva. Tolomeo, Copérnico, Darwin, el movimiento ecológico, Freud, Marx, etc., removieron perspectivas, depuraron perspectivas (Torralba Rosello, 2013).

Hoy no se puede opinar sobre todos los campos del saber, como hicieron los grandes nombres del Renacimiento, pero sí participar de su actitud crítica y de la inquietud intelectual con la que ellos estudiaron los viejos problemas de la cultura (Rico, 1993); estos viejos problemas configuran, hoy, un campo de conocimiento sin fronteras y un racimo de actitudes intelectuales al que solemos denominar humanidades (Lier, 1962). Se equivocarían los que entiendan que ese campo coincide con lo que denominaron los anglosajones humanities o los franceses belles lettres, porque más bien armoniza con aquello a lo que directamente alude la expresión latina studia humanitatis, cuadra con una "materia" que, según Edgard Morin, no puede descuidar en su formación el hombre actual: el estudio de la condición humana (Morin, 2000).

Es evidente que, respecto a la organización convencional de la ciencia, nos situamos en la transversalidad, porque no es el tema de ningún campo de conocimiento convencional, ni siquiera de las ciencias medioambientales. Pero, también es evidente que, para quien se propone pensar en los temas de la formación en entornos humanos, paseando por la biblioteca de Alejandría y no encadenado a la librería de una escuela de pensamiento, la transversalidad es un recorrido que sigue el ejemplo de los humanistas del Renacimiento (Morras Ruiz-Falco, 2000). Entre otras cosas, supone que en vez de buscar un puesto exclusivo, identifiquemos el paisaje incluyente; en vez de abrir brechas segregadoras, tienda puentes en la falla semántica que separa las ciencias y las letras. A esta perspectiva de actividad intelectual John Brockman la llamó "tercera cultura" (Brockman, 1996). Una manera de volver al mundo de la vida donde nació la práctica de la cultura y donde se intuyó el sentido de esa práctica; en el campo abierto donde el hombre se encuentra afanado en el trato con la Tierra como fuente de la vida.

\section{HuMANISMO DE DOMINANCIA}

La perspectiva de estudio en la que fuimos formados durante la mayor parte del siglo xx fue la de un humanismo de dominancia. La reflexión sobre 
el hombre había quedado prendada y prendida, colgada de alguna originalidad humana a la que se concedía la máxima relevancia, algo que distinguiera al humano de todo lo demás. Téngase en cuenta que no se actúa del mismo modo con el resto de los seres vivos. Si por algo se distinguen las especies es por su originalidad. Al construir la taxonomía de los organismos operamos con diferencias y con semejanzas. Cualquier especie es identificada en su originalidad, al tiempo que se inserta en una agrupación más amplia.

Compartimos el acuerdo de que las acciones humanas más características son esas por las que dimos en llamarnos Homo sapiens. Cualquiera de ellas que elijamos, si no quedara informada por un sesgo ideológico nos mostraría una trama que retrocede en el tiempo, que siempre nos ancla en el mundo de la vida, se despliega y conecta hacia atrás y hacia los lados. Esas tramas son difíciles de ver, si la relación con el mundo de la vida no se mira desde una perspectiva cuerpo a cuerpo. Investigar el lugar del ser humano en el mundo encaja con el desvelamiento de los entresijos de la experiencia corporal, encontrar el lugar concuerda con el entendimiento del cuerpo.

Buscar las pautas que nos conectan al mundo de la vida, rechina a irreverente, para algunas personas. Como sonaban a irreverentes las afirmaciones del biólogo español Faustino Cordón: "el hombre es animal genuino" (Cordón, 1982). O como también fue iconoclasta El zoo humano de Morris Desmond (1955). Hoy, sin embargo, advertimos que la correcta relación con la naturaleza se nos plantea, al igual que a las demás especies, como una cuestión de vida o muerte (Attenborou, 1993). La trama de la vida no se rompió al llegar al hombre. Si hemos de hablar, en verdad, de formación y cultivo de un ser humano, de la necesidad que tienen los humanos de cultura, esa necesidad es vitalmente exigible; es decir, los seres humanos necesitan de la cultura para vivir. No se trata de una necesidad supranatural, sino una de nuestras necedades naturales.

El humanismo de dominancia se adhiere a la idea de que la auténtica cultura humana es cultura del espíritu. Cuando el espíritu, la res cogitans, el entendimiento humano, toma el cuerpo como objeto de consideración, ve una máquina, res extensa, un mecanismo de precisión (Koyre, 1973). Entre la perspectiva del cuerpo como reloj y el mundo de los significados de las acciones humanas hay una fractura semántica, que ha dejado a las humanidades a un lado y a las ciencias naturales al otro borde de una quebrada infranqueable. 
Hoy, esa separación entre humanidades y ciencias se hace insostenible por el incremento de problemas éticos que surgen dentro de las prácticas científicas y por la cantidad de incógnitas vitales para las que aún no tienen referencias las humanidades: demuestran una cultura insostenible. La cultura del trato con la corporeidad únicamente fundada en el principio de propiedad -con el cuerpo cada uno puede hacer lo que quiera-, lleva a la proliferación de comportamientos y hábitos de riesgo: una cultura insostenible. Tal vez sea el acceso al código de la vida y el mapeo reciente del llamado código genético lo que haya abierto la puerta a mayor cantidad de problemas para los que no tenemos cultura disponible.

\section{HuMANISMO DE DEPENDENCIA}

Cada vez es más evidente que está "la vida en la cuerda floja”, según titula su libro Niles Eldredge (2001). Describe el autor en el libro "el delta del Okavango", el segmento más sudoccidental del Rift Valley, en el África oriental. Esa depresión está sembrada de lugares famosos de la paleoantropología; en ella se encuentra el desfiladero de Olduvai, donde la pertinacia indagadora de la familia Leakey hizo tan trascendentales descubrimientos; también la región de Turkana o la de Laetoli, donde Mary Leakey encontró dos trazas paralelas de huellas, unas setenta pisadas, con un recorrido de unos 30 metros de longitud, de una familia de australopitecos (Australopithecus Afarensis) y datables en unos 3,6 m. a. (Leakey, 2000). El Okavango es una reliquia de aquellos ecosistemas ancestrales en los que tuvo lugar en diferentes oleadas la historia evolutiva de la especie humana. Ahora, ese ecosistema se encuentra en peligro. "El mismo tipo de historia de amenaza, incluso de colapso inminente, puede contarse de absolutamente todos y cada uno de los ecosistemas del mundo" (Eldredge, 2001, p. 69). Este sentimiento de riesgo de pérdida en el que nos encontramos personalmente implicados es el que produce la energía para alimentar el estudio de la ecología, es el que justifica la necesidad de completar el significado de la educación con el contenido de la educación ambiental y el que plantea el clamor reivindicativo, no solo de un desarrollo sostenible, sino de una "sociedad sostenible", como proyecto colectivo y como criterio 
de valor cultural. Este es el contenido y la intención de fondo de los informes del Worldwatch Institute (USA) y el meollo reivindicativo de los movimientos ecologistas (Brown, 1995).

Los dominios vitales siempre están definidos y marcados por una semiótica con sentido, en la práctica se instituyen como un nodo de relaciones, una encrucijada, entre un específico modo de vida (arquitectura comportamental) y un hábitat determinado. El modo de vida se describe como etograma (sistema de comportamientos) y el hábitat se mapea e identifica en su especificidad por un conjunto multidimensional de variables. La relación entre esas dos complejidades es tan estrecha que causa el hecho de que cada hábitat sea específicamente único; dicho de otro modo, la identidad de la especie se puede describir o por su etograma o por su ecograma, por su autopoiesis o por su ecopoiesis, por su viviendo (modo de vivir) o por su vivienda (modo de habitar).

Hay tantos mundos de vida como especies anden viviendo, tanto percibiendo como conmoviéndose. El concepto de dominio vital tiene un componente de significado espacial y otro de quehacer, de locus-dónde y de lo-que se está haciendo, de contexto de reconocimiento y contexto de padecimiento. Con cada especie extinguida se elimina un capítulo del libro de la vida y una manera de sacar provecho del espacio. Cuesta un poco más calibrar la medida en la que, siendo propios de cada especie, todos esos mundos son interdependientes. Está creciendo la sensibilidad ecológica; esa sensibilidad promueve una cultura ambiental que induce un humanismo de dependencia con el mundo de la vida en su conjunto (García Carrasco, 2004). Desde esta dependencia adquieren nuevas dimensiones los conceptos de calidad de vida, de vida saludable, y se comprende la importancia formativa de traer a las escuelas el Protocolo de Kioto.

Según esto, una segunda orientación de la educación ambiental tiene que ver con enseñar a deliberar sobre el propio modo de vida, sobre el propio dominio vital; no puede comprenderse solo en términos de presente, sino de presente y futuro, de comunidades humanas y de sostenibilidad global del mundo de la vida; en términos de actuaciones y en términos de consecuencias, acciones y consecuencias globales y de largo plazo. Todo ello obliga a la deliberación y al compromiso moral con la salubridad ecológica, con la biológica de la cultura. La meta del sostenimiento de la vida ha de entrar en la deliberación moral; este es el componente moral que implica toda educación ambiental. 
Una tercera orientación de la educación ambiental tiene que ver con la salubridad en el dominio vital personal. La consecuencia fundamental de la transferencia de conocimiento biomédico al modo de vida ha sido la prevención de enfermedades. Pero la prevención del malestar vital incorpora a las deliberaciones el compromiso con la salubridad en los entornos vitales: programas políticos de salubridad social y cultural, la dimensión pública de la salud, que se extiende más allá de la actuación de la medicina.

Buena parte de los problemas sanitarios están relacionados con los patógenos de comportamiento. Entre ellos cabe subrayar los comportamientos personales insalubres (por ejemplo, consumo excesivo de alcohol o de tabaco, la conducción imprudente de vehículos...) y los comportamientos relacionales insalubres (por ejemplo, agresividad y malos tratos en el hogar, incidencia de emocionalidad destructiva...) (Guimon, 2001). Estaríamos ante el ámbito de la salubridad de la vida personal e interpersonal.

Tres aspectos nos parecen especialmente relevantes en la cultura que hemos de tener disponible, para una concepción de la vida saludable en la sociedad de la información: (i) la introducción del concepto de calidad de vida posible en la consideración de todas las formas de disfuncionalidad, de discapacidad o de predisposición de origen genético, como criterio para el trato sociocultural con toda forma de diferencia vital; (ii) las consideraciones socioculturales de trato con la denominada tercera edad y con las actuaciones respecto al bien morir; (iii) el replanteamiento de una ética de la calidad de vida y de trato con la propia corporalidad que confronte con la responsabilidad con la vida, con los estilos de vida y con las prácticas sociales. La calidad de vida implica desarrollar la cultura del espacio como cultura del dominio vital. El discapacitado es el que mejor muestra la dependencia de la calidad de vida respecto de la cultura del espacio.

Si algo caracteriza a los seres humanos, a diferencia del resto de los seres vivos, no es aquella dominancia sobre el mundo de la vida que criticamos, sino el hecho de que la dependencia se les convierte en responsabilidad. Pero demostrar esta dependencia-responsabilidad supone reconstruir la comprensión de la dependencia con la Tierra dentro de un sistema de pensamiento coherente con ella, porque los hombres no reconocemos ni identificamos más que a través de instrumentos conceptuales. Promover educación ambiental es, en definitiva, introducir una manera de hablar del entorno, del mundo de los seres vivos, un modo de incluirnos en esa narración. Dados los riesgos que 
conlleva un planteamiento cultural de dominancia, tendríamos que plantear el concepto de educación insostenible; la disponibilidad de la vida a la que accede el hombre contemporáneo se acrecienta a ritmos tan acelerados desde la ciencia y la tecnología, que incluso nos encontramos, a la hora de afrontar las responsabilidades morales, en estado de cultura indisponible.

\section{HumANismo DE PERTENENCIA}

El encuentro radical con el mundo de la vida aproxima la perspectiva evolutiva a la experiencia vital de la corporeidad; estas perspectivas fueron propuestas en libros como el de Faustino Cordón La naturaleza del hombre a la luz de su origen biológico (1982), o el de Goldstein, que proponía mirar el cuerpo como un todo en sí mismo y como la encarnación de nuestra propia identidad (1961), como nuestra unidad psicosomática.

El humanismo, después de Darwin, se plantea a partir de una idea base: "todos nuestros atributos tan queridamente humanos, lejos de ser únicos, están enraizados en nuestra historia evolutiva de primates" (Bradshaw, 2003). Este pedagógico los filósofos lo califican de heurístico, y supone volver al origen y desembarazarnos, la deconstrucción de buena parte de la superestructura cultural, que nos hace ver los caracteres genuinos que nos identifican como especie.

Estudiando el cuerpo y rastreando sus coherencias con los antecesores en la estirpe, nos topamos con las evidencias de la unidad del mundo de la vida. Lo que Ernst Haeckel en 1866 denominaba "la raíz común de los organismos" otros lo llaman "la trama de la vida" (Capra, 1998), "la pauta que conecta" (Bateson, 1990), "la hebra común que pasa a través de toda la existencia biológica" (Fortey, 1999). Todas las expresiones de la vida, desde los organismos unicelulares al hombre comparten características comunes en el nivel celular. Por aquí encontraríamos un humanismo de pertenencia. Este humanismo de pertenencia debe mostrar que es precisamente nuestro modo biohumano de ser el que sustenta nuestro modo etohumano de comportarnos, de reconocernos, de querernos y de entendernos, mediante grandes dosis de emoción y pensamiento: es el humanismo que nos permite decir la verdad acerca de que somos el único ser vivo que es capaz de hacerlo así y de contarlo de esta manera. La 
verdad de todo lo demás depende de lo que nosotros nos digamos acerca de cómo somos y cómo nos vemos.

La primera respuesta a la pregunta ¿qué es un hombre? no puede dejar de ser, pues, que los seres humanos forman una especie animal dentro del mundo de los seres vivos; en los albores del siglo XXI, sin "ponerle peros". Y esto por varias razones:

- Para que lo que se piense de sí mismo y sobre los demás se haga coherente con nuestras necesidades primarias (necesidades de cuidado, alimentación, lugar donde vivir...), con el sistema de comunicación básico (apariencia, comunicación corporal, contacto...), con el hecho de tener un historial vital (infancia, adolescencia...vejez), con la corporalidad como desencadenante de valoración (emoción, afectividad...), con la pertenencia de todos a una especie única (aceptación de las diferencias...).

- Para que, al definir el modo cultural de tratar con la Tierra, con las demás especies vivientes y entre nosotros, pase a primer plano el hecho de que el hombre pertenece a la misma trama de la vida que el resto, que también a él le alcanza la pauta y patrón que los mete a todos dentro del grupo de los seres vivos, con un destino global común; ya no se trata de dominancia, ni siquiera de dependencia, sino de auténtica pertenencia; este ha de ser un objeto de atención y de admiración.

- Este planteamiento deja asentados los criterios para una educación ambiental: que acompañe a la socialización, a la cultura de la comunicación, a la cultura emocional y a los proyectos de construcción y transformación del paisaje y del entorno vital; una cultura ambiental que acompañe a la cultura científico-técnica, a la cultura estética y a la cultura ética.

- Para que las humanidades se reconstruyan desde dentro del conocimiento y la indagación sobre el mundo de la vida, en la confluencia de las ciencias y las letras, donde hermanar en una responsabilidad común la sostenibilidad; para que las generaciones futuras tengan las mismas oportunidades de calidad de vida que hicieron posible la aparición de nuestra especie. Esto es cambiar de "macroscopio", cambiar de perspectiva.

Un ejemplo de este modo de reflexión humanística en tercera cultura es la que comparten Changeux y Ricoeur en la obra Lo que nos hace pensar, la naturaleza y la regla (1999). Sin esta forma de humanismo, sin la afición cultural 
a caminar sobre estos temas que cruzan las fronteras verticales de las ciencias y las letras, sin andar en estos temas y sin emocionarse con ellos, no se puede exigir al profesorado que haga con sus alumnos ejercicios de "transversalidad"; cada nueva exigencia cultural no tendrá otra salida que una nueva asignatura para el currículo. En diversos ámbitos se indica que la transversalidad ha fracasado. No fracasa porque no estuviese suficientemente justificada, sino porque para hacerse posible tenemos los docentes que modificar la perspectiva desde la que estudiamos y la perspectiva desde la que enseñamos. Puede que la perspectiva analítica de fragmentación sea estrategia aconsejable para poder investigar en la frontera. Pero, para formar la mente de un ser humano que no está situado en la frontera de la ciencia sino en las circunstancias de la vida es preferible ver el mundo como un todo.

\section{A MOdO DE CONCLUSIÓN: MACROSCOPIOS PARA VER LA VIDA DESDE LOS SERES HUMANOS}

Los laboratorios actuales se encuentran llenos de complejos y sofisticados instrumentos. Por encima de los instrumentos y de los datos se encuentran los "planteamientos" intelectuales, las perspectivas dentro de las cuales los datos se instituyen y se transforman en prueba. Los principales instrumentos intelectuales están constituidos por herramientas mentales, entre las que desempeñan un papel de primer orden los modelos, las analogías, las metáforas. Los "modelos" funcionan como "macroscopios", en el sentido de que proporcionan materiales con los que construir nuestras propias interpretaciones: "vemos" a través de ellos, son como el cristal que "corrige" y modifica, aumentan o complementan, la imagen del mundo que vamos construyendo, son instrumentos del pensamiento: instrumentos psicológicos.

\section{1 ¿Con qué materiales construimos los macroscopios?}

Los materiales de construcción de perspectivas consisten siempre -aunque se encuentren ocultos dentro de la carcasa de la convicción, la argumentación o 
las evidencias- en un conjunto de supuestos básicos, sistemas de proposiciones aceptadas, compromisos intelectuales compartidos, familiaridades y afiliaciones (Pérez Ransanz, 1993). No estamos solo ante un material de información: esos núcleos constituyen auténticos instrumentos para la comprensión; contienen supuestos y compromisos básicos en ontología (qué creemos que son y cómo son los objetos, comunidades de personas, seres vivos, cosas o procesos de los que hablamos), generalizaciones simbólicas o leyes fundamentales aceptadas (a qué reglas, principios o normas obedecen), procedimientos y técnicas de investigación (cómo pueden ser lógicamente estudiados, con qué materiales construir sus modelos teóricos), criterios de evaluación (cómo definir criterios de calidad de acción o de proceso, cuándo tener seguridad de que la situación y la acción creada está en la orientación de la meta) y criterios de corroboración, dentro de los cuales reconocemos formas ejemplares de plantear problemas y de solucionarlos. La concreción de todos estos elementos constituye la matriz disciplinar para la actividad de conocimiento y proporciona el criterio desde el cual elaborar los "textos" de las disciplinas académicas. Cuando una perspectiva es compartida, la reflexión y la acción acorde con ella se califican de "normales" (Kuhn, 1971); algunos prefieren hablar de "discurso dominante", porque el discurso dentro de la perspectiva pretende autoabastecerse y autoalimentarse, se construye precisamente con la intención básica de autosostenerse y reproducirse con privilegios (García Carrasco y García-Peñalvo, 2003).

Dentro de la permanente corriente de impresiones sensoriales que recibimos, la perspectiva nos proporciona el criterio y guía de a dónde dirigir la atención. Ya los propios receptores sensoriales tienen claves acerca de lo que considerar novedad y, en correspondencia, qué será lo que produzca sorpresa; la perspectiva nos proporciona claves acerca de dónde buscarla y a través de qué seguros caminos. La perspectiva crea el marco dentro del cual queda a la expectativa de la dificultad para la interpretación o la solución del problema, pues los datos se organizan como problema dentro de la perspectiva. Sobre todo, es en el interior de la perspectiva donde la forma de comprensión del problema, la decisión metodológica, adquirirá el cometido de lazarillo para la solución.

Cambiar de perspectiva es como cambiar de vivienda: supone un traslado de la subjetividad de un mundo de significados a otro. 


\subsection{Sociofilia y biofilia}

La teoría de la educación, la reflexión sobre la formación, la hemos construido dentro de un contexto de sociofilia, poniendo especialmente de manifiesto que tiene lugar en lo que denominamos medio socializante cementado por la comunicación lingüística. Tal como los entendemos, los procesos de socialización solo se dan en la acción comunicativa y despliegan su fuerza formativa en un medio de procesos de entendimiento y decisiones (que en las personas adultas de referencia se asocian además a razones internas): como las hortalizas que vemos crecer y cortamos desde la superficie de la tierra hacia arriba, menospreciando las raíces. Entre los elementos fundamentales de la socialización se encuentra no solo el aspecto de modelado, sino también el proceso revisionario por el que cada cual puede reconstruir su proyecto de vida, un ejercicio de autonomía. Entendemos la educación como un proceso de construcción y de revisión de identidades entre gente humana.

Tanto el proyecto de progreso cultural en conjunción con la sostenibilidad de la biosfera, como el de progreso científico tecnológico en conjunción con la sostenibilidad de la condición humana, nos exigen el planteamiento de una teoría de la educación que requiere del añadido de una actitud de biofilia. Denominamos biofilia, con O. Wilson, a la "tendencia innata de dirigir nuestra atención a la vida y a los procesos vitales" $(1989$, p. 9). En la evolución mental de los seres humanos desempeñó un papel fundamental la afinidad con los vitalmente semejantes y el fomento de la creatividad de sobrevivencia, una cultura para vivir, la ventaja de poder convertir la vida en proyecto.

Creo que esta actitud de biofilia tiene dos direcciones: la de retroceder en el tiempo para identificar arqueológicamente la humanidad insoslayable y radical y la de ampliar el campo de la experiencia para incluir en ella la diversidad de las formas de vida con las que compartimos estructura, dependencia y pertenencia: el camino por donde vinimos a la vida.

\subsection{El intercambio subjetivo, el núcleo de la noción de cultura, como carácter etológico de la especia humana}

El etograma de los humanos incluye predisposiciones específicas hacia la relación asimétrica (adulto-niño, experto-aprendiz); es decir, el etograma humano 
incluye comportamientos intersubjetivos orientados a promover el aprendizaje. Nacemos con todos los dispositivos de "curiosidad" necesarios y en permanente estado de actividad de rastreo del entorno, para que, en el entorno social adecuado, la mente pueda activarse a rendimiento pleno. Un indio yanomami, un bosquimano, no es un "deficiente", ni un humano anormal, ni un "hombre salvaje"; las diferencias culturales entre ellos y los profesionales de las grandes industrias o los investigadores de las selectivas universidades no estriban en los modos del funcionamiento de la mente (cerebro), sino en las prácticas a las que aplican sus capacidades mentales. Quizás, en algunas disposiciones mentales, como la de comprender la intención del otro, la de corresponder selectivamente a compromisos de afiliación en la práctica compartida de sobrevivencia, o en la de contribuir a la exigencia de amparo emocional a las crías, encontramos entre aquellas mayores calidades de relación, por encontrarse antropológicamente menos descoyuntados por dinámicas coincidentes en el tiempo, al tiempo que divergentes en lo que reclaman. A veces, encontramos grupos humanos de culturas menos sofisticadas en estados menos estresados y, por lo tanto, más disponibles para el sostenimiento de la urdimbre intersubjetiva. La primera premisa de una teoría de la educación incluye enunciados referidos al hecho de que la exigencia de situaciones-acciones de formación les llegan a los humanos por vía evolutiva y no por creación cultural.

Las exigencias de educación formal (de preparación ocupacional) son tan grandes en las sociedades complejas, que ocultan las necesidades vitales de conformación personal dependientes de relaciones intersubjetivas y de vínculos sociales. Tal es el significado fundamental de la necesidad de vínculo afectivo y el significado fundamental del sentimiento de pérdida o duelo.

La cultura en los humanos, en el meollo, es una posibilidad y una necesidad de más vivencia. Lo demuestran varios hechos: (i) la gravedad del riesgo vital asociada al abandono y al aislamiento; (ii) los riesgos asociados a las carencias relacionales básicas en edades tempranas; (iii) los riesgos en la calidad de vida asociados a prácticas empobrecedoras de las relaciones interpersonales y escatimadoras de tiempo disponible para el disfrute de la pegajosidad biológica y la vida en proximidad. La disponibilidad de tiempo para nosotros no es exclusivamente una demanda infantil (p. e. el "juega conmigo", "ven conmigo", "llévame", "estate aquí"), es una demanda que va cambiando de forma y que tiene dos máximos: uno al principio del trayecto vital y otro al 
final; precisamente, los dos momentos en los que el riesgo de abandono, de aislamiento o de mal trato se presenta como amenaza.

Las competencias en el dominio del entorno evolucionaron en coderiva con el aumento de competencia social; el conocimiento sobre el mundo fue a la par del desarrollo de competencias sociales. Al aumentar la competencia técnica en el manejo del entorno de sobrevivencia (inteligencia técnica), la especie humana reducía la necesidad de andar alerta, de quedar absorta en encontrar medio de vivir y escamotear el riesgo de sucumbir al depredador (inteligencia natural); esto proporcionó tiempo para dedicarse a más-vivir (inteligencia social). El desarrollo de la inteligencia social, la resolución de problemas sociales, poniéndose en el lugar del otro ("teoría de la mente"), probablemente se encuentra en el origen de la anticipación de consecuencias necesarias al desarrollo de la creatividad técnica. De ahí, el riesgo de que la superestructura de formación derivada de la sustentación del sistema económico y científico tecnológico, el "más vivir de la gente", del que hablaba Ortega y Gasset, deje inerme y a la intemperie las necesidades de la "más-vivencia de la gente".

Decía Azorín que "vivir es ver volver", reflexionar es intentarlo. Para recuperar el sentido profundo de cultura no nos basta ya con volvernos de nuevo al mundo rural, sino mirar a través de la costra de la civilización, por debajo de la epidermis de las razas, descarnando las prácticas humanas de sus complicados artefactos, hasta encontrar el punto en el que aparece nuestra común pertenencia al mundo de la vida. No hay que descomponer, sino retropensar, vernos en la forma en la que hemos pasado la mayor parte de nuestra historia evolutiva de Sapiens, en los tiempos de la Eva negra, hace por lo menos dos cientos mil años.

\section{BiBLIOGRAFÍA}

Attenborou, D. (1993). La vida a prueba. Barcelona: Ed. B de Divulgación Científica RBA.

BAtANer, E. (2001). La percepción pública de la ciencia. Salamanca: Ed. Universidad de Salamanca.

Bateson, G. (1990). Espíritu y naturaleza. Buenos Aires: Amorrortu. 
Bermudez de Castro, J. M. (2010). La evolución del talento. Barcelona: Random House Mondadori.

Bradshaw, T. (2003). Évolution humaine. Une perspective neuropsychologique. Bruxelles: De Boeck.

Brockman, J. (1996). La tercera cultura. Más allá de la revolución científica. Barcelona: Metatemas / Fundación Caixa.

Brown, L. (1995). L'état de la planète 1995-1996. París: La Découverte.

CAPRA, F. (1998). La trama de la vida. Una nueva perspectiva de los sistemas vivos. Madrid: Anagrama.

Changeux, J. P. (1986). El hombre neuronal. Madrid: Espasa Calpe.

Changeux, J. P. y Ricoeur, P. (1999). Lo que nos hace pensar. La naturaleza y la regla. Barcelona: Península.

Cordón, F. (1982). La naturaleza del hombre a la luz de su origen biológico. Barcelona: Antropos.

DAmasio, A. R. (1996). El error de Descartes. La emoción, la razón y el cerebro humano. Barcelona: Editorial Crítica-Drakontos.

Darwin, C. (2003). El origen de las especies. Madrid: Alianza Editorial.

De Rosnay, J. (1977). El macroscopio: hacia una visión global. Madrid: AC.

DehaEne, S. (2014). El cerebro lector. Últimas noticias de las neurociencias sobre la lectura, la enseñanza, el aprendizaje y la dislexia. Argentina: Siglo XXI Editores.

Dehaene, S. (2019). ¿Cómo aprendemos? Los cuatro pilares con los que la educación puede potenciar los talentos de nuestro cerebro. Argentina: Siglo XXI Editores.

Dobzhansky, Th. (1937). Genetics and the origin of species. Nueva York: Columbia University Press.

Eldredge, N. (2001). La vida en la cuerda floja. La humanidad y la crisis de la biodiversidad. Barcelona: Metatemas.

FORTEY, R. (1999). La vida, una biografía no autorizada. Una historia natural de los primeros cuatro mil millones de años de vida sobre la Tierra. Madrid: Taurus.

García CARrasco, J. (2004). La comprensión de la vivienda como un dominio vital de los humanos. Revista Española de Pedagogía 62(288), 229-256. 
García Carrasco, J. y Garcia-Peñalvo, F. (2003). Marco de referencia pedagógico en el contexto informacional. Bordón. Revista de Pedagogía 4(54), 527-545.

Goldstein, K. (1961). La naturaleza humana a la luz de la psicopatología. Buenos Aires. Paidós.

Guimon, J. (2001). Salud mental relacional. Concepto, etiología y diagnóstico. Madrid: Core Academic.

Jonas, H. (1995). El principio de Responsabilidad. Ensayo de una ética para la civilización tecnológica. Barcelona: Herder.

Koyre, A. (1973). Du monde clos à l'univers infini. París: Gallimard.

Kunn, T. S. (1971). La estructura de las revoluciones cientificas. México: Fondo de Cultura Económica.

LEAKey, R. (2000). El origen de la humanidad. Madrid: Debate.

LIER, H. (1962). Le nouvel age. París: Casterman.

Lier, H. (1965). Les humanités du XX $x^{\mathrm{e}}$ siècle. París: Casterman.

Morin, E. (2000). La mente bien ordenada. Repensar la Reforma. Repensar el pensamiento. Barcelona: Seix y Barral.

Morras Ruiz-Falco, M. (2000). Petrarca, Bruni, Valla, Pico de la Mirándola, Alberti. Manifiestos del Humanismo. Barcelona: Península.

Morris, D. (1995). El zoo humano. Barcelona: Editorial Plaza \& Janés.

Mosterín, J. (2001). Ciencia viva. Reflexiones sobre la aventura intelectual de nuestro tiempo. Madrid: Espasa.

Pérez Ransanz, A. R. (1993). Modelos de cambio científico, en C. U. Moulines (ed.), La ciencia: estructura y desarrollo (pp. 181-203). Madrid: Trotta.

Rico, F. (1993). El sueño del humanismo. De Petrarca a Erasmo. Madrid: Crítica.

SÁnchez Ron, J. M. (2011). La Nueva Ilustración: Ciencia, Tecnología y Humanidades en un Mundo Interdisciplinar. Oviedo: Ediciones Noberl.

SCHELER, M. (1979). El puesto del hombre en el Cosmos. Buenos Aires: Losada.

Snow, C. P. (1977). Las dos culturas y un Segundo enfoque. Madrid: Alianza Editorial.

Torralba Rosello, F. (2013). Maestros de la sospecha. Marx, Nietzsche, Freud. Barcelona: Fragmenta Editorial.

Willson, E. O. (1989). Biofilia. México: Fondo de Cultura Económica. 